\title{
Data acquisition and real time signal processing of plasma diagnostics on ASDEX Upgrade using LabVIEW RT
}

\author{
L.Giannone $^{\mathrm{a}}$, M.Cerna ${ }^{\mathrm{b}}$, R.Cole ${ }^{\mathrm{c}}$, M.Fitzek $^{\mathrm{c}}$, A.Kallenbach $^{\mathrm{a}}$, K.Lüddecke ${ }^{\mathrm{c}}$, P.J.McCarthy ${ }^{\mathrm{d}}$, A.Scarabosio ${ }^{\mathrm{a}}$, \\ W.Schneider ${ }^{\mathrm{a}}$, A.C.C.Sips ${ }^{\mathrm{a}}$, W.Treutterer ${ }^{\mathrm{a}}$, A.Vrancic ${ }^{\mathrm{b}}$, L.Wenzel ${ }^{\mathrm{b}}$, H.Yi $^{\mathrm{b}}$, K.Behler ${ }^{\mathrm{a}}$, T.Eich ${ }^{\mathrm{a}}$, H.Eixenberger ${ }^{\mathrm{a}}$, \\ J.C.Fuchs ${ }^{\text {a }}$, G.Haas ${ }^{\mathrm{a}}$, G.Lexa ${ }^{\mathrm{a}}$, M.Marquardt ${ }^{\mathrm{a}}$, A.Mlynek ${ }^{\mathrm{a}}$, G.Neu ${ }^{\mathrm{a}}$, G.Raupp ${ }^{\mathrm{a}}$, M.Reich ${ }^{\mathrm{a}}$, J.Sachtleben ${ }^{\mathrm{a}}$, \\ K.H.Schuhbeck ${ }^{\mathrm{a}}$, T.Zehetbauer ${ }^{\mathrm{a}}$, S.Concezzi ${ }^{\mathrm{b}}$, T.Debelle ${ }^{\mathrm{b}}$, B.Marker $^{\mathrm{b}}$, M.Munroe $^{\mathrm{b}}$, N.Petersen ${ }^{\mathrm{b}}$, D.Schmidt $^{\mathrm{b}}$, NI Big \\ Physics Team ${ }^{\mathrm{b}}$, ASDEX Upgrade Team ${ }^{\mathrm{a}}$ \\ ${ }^{a}$ Max-Planck-Institut für Plasmaphysik, EURATOM-IPP Association, \\ D-85748 Garching, Germany \\ ${ }^{b}$ National Instruments, Austin, TX 78759-3504, Texas, USA \\ ${ }^{c}$ Unlimited Computer Systems GmbH, 82393 Iffeldorf, Germany \\ ${ }^{d}$ Department of Physics, University College Cork, Association EURATOM-DCU, Cork, Ireland
}

\begin{abstract}
The existing VxWorks real time system for the position and shape control in ASDEX Upgrade has been extended to calculate magnetic flux surfaces in real time using a multi-core PCI Express system running LabVIEW RT 8.6. Real time signal processing of bolometers and manometers is performed with the on-board FPGA to calculate the measured radiated power flux and particle flux respectively from the raw data. Radiation feedback experiments use halo current measurements from the outer divertor with real time median filter pre-processing to remove the excursions produced by ELM's. Integration of these plasma diagnostics into the control system by the exchange of XML sheets for communicating the real time variables to be produced and consumed is in operation. Reflective memory and UDP are employed by the LabVIEW RT plasma diagnostics to communicate with the control system and other plasma diagnostics in a multi-platform real time network.
\end{abstract}

Key words: tokamak control, data acquisition, bolometer, manometer, halo current, FPGA, LabVIEW RT,

\section{Introduction}

The LabVIEW RT operating system is capable of dealing with large scale data acquisition and control problems. The data acquisition and control system of European Southern Observatory's planned European Extremely Large Telescope is the subject of a feasibility study [1]. The telescope's primary active mirror will be $42 \mathrm{~m}$ in diameter with 984 hexagonal mirror segments. All segments must be in strict alignment continuously, even in windy conditions. The control system must respond to a total of 6,000 sensor inputs and then send control signals to 3,000 actuators within the inputoutput cycle of $1 \mathrm{~ms}$ to maintain mirror segment alignment. It has also been chosen as the platform for controlling the positions of collimators of the particle beam in the Large Hadron Collider in CERN [2]. The FPGA modules control approximately 600 stepper motors with millisecond synchronization over the $27 \mathrm{~km}$ length of the accelerator. In fusion experiments, LabVIEW RT Preprint submitted to Fusion Engineering Design has also been used to calculate in real-time the plasma density profile $(10 \mathrm{~ms})$, the Shafranov shift $(10 \mathrm{~ms})$, the plasma vertical and horizontal position $(20 \mu \mathrm{s})$ and to control the plasma shape $(1 \mathrm{~ms})$ in the TEXTOR tokamak [3]. Standard off-the-shelf components instead of in house designs were used to build these data acquisition and control systems.

There are five LabVIEW RT data acquisition systems for real time signal processing of plasma diagnostic measurements in operation on ASDEX Upgrade. The details of each data acquisition system and the real time signal processing of the measurements for communication to the control system are discussed in the following sections. In section 2, the extension of previous work concerning real time flux surface reconstruction from magnetic probes measurements and the currents in the poloidal and toroidal field coils is presented. The bolometer and manometer data acquisition systems take advantage of off-the-shelf FPGA technology and July 5,2010 
are discussed in sections 3 and 4 respectively. Halo current and loop voltage measurements are detailed in section 5.

The real time control system of ASDEX Upgrade employs a UDP based framework for command control of the plasma diagnostics. These commands initialize the exchange of information between the plasma diagnostics and control system via XML files to coordinate the details of their operation. This information consists of a list of the real time variables to be produced and consumed by each diagnostic and whether this data will be communicated by UDP or by reflective memory. The essential software to connect the VxWorks control system [4], Solaris based real time plasma diagnostics [5] and LabVIEW RT real time plasma diagnostics is available for each operating system. Specifically, UDP communication in LabVIEW RT is available as part of the Professional Development System installation. The parsing of XML files was realized by a third party package [6] and allows the XML files to be read and written in the graphical development environment of LabVIEW. On KSTAR, EPICS is used as the middle-ware to integrate the LabVIEW diagnostics into the plasma control system [7].

The graphical development environment of LabVIEW is an alternative to a text based open source development environment for creating multithreaded real time applications. The LabVIEW RT real time data acquisition application consists of three parallel executing loops. The UDP commands are continuously monitored in the primary loop. The data acquisition loop waits for notification of the time chosen as a start trigger and then writes a data file for FTP transfer to the archiving software upon completion. The real time communication loop sends data via UDP or reflective memory to the control system and other plasma diagnostics, depending on the configured mode. The data to be sent to the control system by the communication loop are transferred from the data acquisition loop by real time FIFO's.

\section{Magnetic equilibrium}

Measurements of magnetic field from magnetic probes and saddle coils, currents in the poloidal and toroidal field coils and halo currents to the divertor are the set of 100 measurements usually processed offline with a tokamak equilibrium code to reconstruct the magnetic flux surfaces in ASDEX Upgrade [9]. This data set is sampled simultaneously with $10 \mathrm{kHz}$ for the duration of the $10 \mathrm{~s}$ discharge using 16 PXI 6143 data acquisition cards mounted in an 18 slot PXI 1045 chassis and connected via a PXI-PCIe 8361 bridge to a PCIe
LabVIEW RT 8.6 system with two Intel Xeon $3.0 \mathrm{GHz}$ quad core processors [10]. It was shown that the magnetic probe and flux loop measurement prior to a discharge could be monitored for sensor or integrator failure in real time.

This earlier work has been extended by calculating the response matrix of the magnetic probes and flux loops to current elements on a grid with $4 \mathrm{~cm}$ spacing in the vertical and horizontal directions. These stored response matrix values are then used to calculate the expected magnetic probe and flux loop signals resulting from an axisymmetric current filament carrying the plasma current at the position closest to the magnetic axis in real time [11]. This calculation is sufficient to monitor the magnetic probe or flux loop measurement to identify a sensor or integrator failure during a discharge.

A function parameterization algorithm rather than the off-line Grad-Shafranov algorithm is used to calculate the tokamak equilibrium flux surfaces on a $39 \times 69$ grid for real time control $[12,13]$. The 95 plasma position and shape parameters are also calculated in real time. Currently, the magnetic flux surfaces are available with a $6 \mathrm{~ms}$ cycle time and the plasma position and shape parameters are available with a $1.5 \mathrm{~ms}$ cycle time. The inclusion of plasma current profile information from the MSE diagnostic will impose additional constraints to the equilibrium reconstruction and improve accuracy. However, this requires increasing the number of principal components from 20 to 22 with 18 principal components for the 60 magnetic probe and flux loop measurements and four principal components for the nine MSE measurements. Therefore the number of principal components and their quadratic terms increases from 231 to 276 and the matrix multiplication to calculate the 2691 values of the poloidal flux on the $39 \times 69$ grid is proportionally slower. The real time communication to the control system of the poloidal flux values is an important component of planned experiments concerning real time NTM stabilization and disruption mitigation on ASDEX Upgrade [4]. The contours in poloidal flux on the grid are the magnetic flux surfaces of the plasma equilibrium.

The communication between the magnetics LabVIEW RT data acquisition system and the VxWorks control system prior to the discharge and during the real time phase now uses the fiber optic cable connecting the reflective memory cards installed in a PCI slot in each system. The measured average latency for the DMA transfer of the 2691 values of the poloidal flux matrix calculated by the function parameterization algorithm is less than $1 \mathrm{~ms}$. An average latency of $20 \mathrm{~ms}$ was mea- 
sured using UDP to transfer this array over the Gigabit Ethernet connection. In contrast, the transfer of smaller packets containing one or two values from the diagnostics discussed below using UDP had a measured average latency of less than $250 \mu \mathrm{s}$. This clearly demonstrates that reflective memory is a possible choice for distributing large arrays in real time between nodes independent of operating system. Preliminary tests of a Dolphinics DXH 510 PCIe reflective memory card using the standard Linux driver indicates that this performance can be improved by an order of magnitude [14].

It is envisaged that the calculation of tokamak equilibria by the Grad-Shafranov equation could run with a cycle time of a few milliseconds on a multi-core CPU system with GPU coprocessor $[15,16]$. A non-linear Grad-Shafranov solver with 7th order arbitrary polynomial on the right hand side on a $63 \times 111$ grid completes a single iteration in $250 \mu$ s on a multi-core CPU. Convergence to a solution in four iterations with a relative error of $10^{-5}$ has been demonstrated. When fully tested, this solver would be an alternative to the solver employed by the real time version of EFIT [17]. This real time version of EFIT is currently widely used for real time control of tokamaks.

\section{Bolometer}

The temperature increase of the bolometer foil, resulting from heating of the four micron thick platinum absorber by radiated power from the plasma, is measured as a voltage difference on a Wheatstone bridge [18] using the AD7730 (Analog Devices) bridge amplifier. A $5 \mathrm{~V}$ square wave at $2 \mathrm{kHz}$ is applied to the Wheatstone bridge arrangement of a bolometer foil [19]. The measurement of 32 bolometer bridges in a 19 in. rack module has been realized. The digitized signals of up to 128 channels are transferred serially to a single PXI 7813R FPGA card with 4 x 40 DIO connectors from four modules. The raw signal from four bolometer channels during a discharge is shown in Fig 1. In comparison with the AC system previously described [18], the offset compensation is greatly simplified. The AD7730 has several features including the ability to correct the DC bridge offset due to a mismatch of the meander resistances, ratiometric measurement of the bridge voltage and square wave excitation which avoids complications arising from cable capacitance when using AC excitation. The next stage of development foresees setting the individual gains of the ADC's by reading a XML file generated by a dedicated web page.

The absorbed power of the bolometer foil, which is a weighted sum of the raw data and its filtered time derivative, is calculated in real time on the FPGA. The differentiating filter for the FPGA was generated using the LabVIEW Digital Filter Design Toolkit. Three calibration factors are needed to convert the bolometer signal output of the Wheatstone bridge to an incident power. These are the normalized heat capacity, $\kappa$, the cooling time constant, $\tau$ and the meander resistance at the operating temperature of the foil, $\mathrm{R}_{\mathrm{OH}}$. The procedure and equipment used for the calibration are detailed elsewhere ( see equations A16 and A14 for the definitions of $\kappa$ and $\tau$ ) [20]. The incident power to the measurement foil is then given by :

$$
P_{\text {rad }}=\frac{2}{U}\left(R_{O H}+2 R_{C}\right) \kappa\left[\tau \frac{d u_{d}}{d t}+u_{d}\left(1-\frac{U^{2}}{4 \kappa\left(R_{O H}+R_{C}\right)^{2}}\right)\right](1)
$$

where $\mathrm{P}_{\text {rad }}$ is the incident power absorbed by the measurement foil, $\mathrm{U}$ is the amplitude of applied square voltage, $\mathrm{u}_{d}$ is the amplitude of the measured signal and $\mathrm{R}_{C}$ is the cable resistance. The calibration coefficients are measured prior to each discharge. The raw data are processed using Equation 1 to calculate the power flux to each of the 128 bolometer foil absorbers in real time. The radiated power measured by four bolometers during a discharge is shown in Fig 2. Four lines of sight, representative of radiation in the plasma core and plasma edge, are summed on the FPGA. The ratio of these two values is the radiation peaking factor and is also calculated in real time. If a set radiation peaking factor is exceeded, the control system responds by activating central ECRH deposition in the discharge to mitigate impurity accumulation [21]. This solution replaces the direct connection of two analogue signals to the control system from single lines of sight viewing the plasma core and plasma edge. The new solution provides a more robust peaking factor of the radiation profile, as it is less sensitive to the exact position of the magnetic axis.

Real time tomography is being integrated into the data acquisition application. Tomographic reconstructions with a cycle time of $2 \mathrm{~ms}$ on simulated data have been demonstrated. The cycle time should be further reduced as multi-core versions of this algorithm become available.

\section{Manometer}

The measurement of the ion current resulting from the ionization of neutral particles by electrons emitted from a hot filament is the basis of the manometer neutral flux measurement on ASDEX Upgrade [22]. This ionization gauge measures a neutral particle flux at various spatial locations and makes a vital contribution to scrape 
off layer modeling required for predicting ITER operation [23]. However, in a fusion environment specialized electronics is required to feedback regulate the emitted electron current, provide adjustable gain for the ion current, modulate the applied potentials to the ionization gauge and demodulate the measured electron and ion current. The electronics for four ionization gauges uses eight analog 16 bit inputs with a $-10 \mathrm{~V}$ to $10 \mathrm{~V}$ input range, four analog outputs and ten DIO outputs of a PXI 7852R FPGA card for this purpose. The PID control of the electron current emission of a filament uses a module from the FPGA software library to calculate the heating filament current required to maintain the $200 \mu \mathrm{A}$ set point. The raw signals at the ADC for electron and ion currents acquired at $200 \mathrm{kHz}$ are shown in Fig. 3. An overview of the operation of the ionization gauge in a discharge is shown in Fig 4. The chopped electron and ion current signals are demodulated on the FPGA in real time to provide the required subtraction of background signal. The particle flux is calculated using a look up table of the ratio of ion and electron current versus particle flux that is produced in dedicated calibration discharges. The neutral gas pressures of four ionization gauges are acquired and calculated in real time on each FPGA card at up to $10 \mathrm{kHz}$. An optical MXI-4 PCI bus extender is used for remote data acquisition, as the ionization gauge electronics must be located inside the experimental hall. Remote control of the power supplies is by RS-232 bus, and the gain factors of the individual ion current measurements are controlled by DIO lines of the FPGA card. It is also planned to set these gain factors by a dedicated web page. This web page will create a XML file for interfacing to the data acquisition system. The final stage of operation will have 20 ionization gauges connected to five FPGA cards at three remote data acquisition stations. The real time measurement of particle flux by the manometer is used by the control system to set the gas pressure in the vacuum vessel prior to initiating the plasma discharge.

\section{Halo current and loop voltage}

Impurity gas puffing of nitrogen is necessary in high power operation on ASDEX Upgrade to protect the tungsten coating on the divertor tiles from high heat load damage by increasing the radiated power. This was necessary because intrinsic impurities were reduced by the completion of tungsten coating on all plasma facing components [24]. The power load to the divertor is monitored by measuring the current to the inner and outer divertor tiles. Feedback control of the radiated power level is achieved by impurity seeding. The magnitude of the divertor tile current depends particularly on the peak electron temperature in front of the outer target. The divertor tile current is measured by a shunt connected to an isolating amplifier (Analog Devices AD 215 ) and 2 PXI 6143 digitizers sampling at $100 \mathrm{kHz}$. Between ELM's, the current is driven by the electron temperature difference of the outer and inner divertor plasma. This shunt current has been compared to thermography measurements of the divertor tile temperature and shown to be approximately proportional to the peak power load to the outer target. The divertor temperature is therefore regulated by adjusting the flux of impurity gas to increase radiated power in the divertor. The outer divertor tile current and power load to the divertor necessary for safe operation is then achieved.

However, the divertor tile current and power load are both modulated by ELM's, as shown in the left diagram of Fig. 5. A median filter over the previous 700 points is calculated every millisecond in real time to remove the effects of ELM's from the divertor tile current measurements. The total outer divertor current is calculated as a weighted sum of the 100th largest value of current of three individual tiles. In the right diagram of Fig. 5, the ELM free current (red) is feedback regulated by nitrogen gas puffing to the set value of three (dashed purple). The smallest current value found by the median filter is not used, as negative current spikes measured when the divertor begins to detach should be avoided. This solution became necessary as sampling at $100 \mathrm{kHz}$ was not possible on the analogue measurement connected directly to the control system. The median filtering of the ELM peaks on the divertor tile current required this higher sampling rate.

Loop voltages and passive stabilizer loop voltages to ground are measured by direct connection to $16 \mathrm{DMM}$ 4072 PXI modules in a PXI 1045 chassis with a sampling rate of $30 \mathrm{kHz}$ and resolution of 16 bits. These modules are rated at $300 \mathrm{~V} \mathrm{DC}$ isolation voltage. These commercially available modules increased the measurement bandwidth that was limited by the earlier used isolation amplifiers.

\section{Conclusion}

There are five LabVIEW RT data acquisition systems for real time signal processing of plasma diagnostic measurements in operation on ASDEX Upgrade. They are integrated into the VxWorks control system real time communication framework by the exchange of XML files. Processed data are communicated to the control system and other plasma diagnostics in real 
time by fiber optic cables using either UDP or reflective memory. The 2691 values of the poloidal flux matrix representing the tokamak magnetic equilibrium are transferred using reflecting memory in under $1 \mathrm{~ms}$ with a 6 ms cycle time.

The calculation of magnetic flux surfaces in real time takes advantage of the graphical development environment of LabVIEW to produce a real time multithreaded application utilizing multi-core processors. The bolometer and manometer diagnostics employ digital and analog inputs in combination with FPGA's to realize compact real time diagnostics. Remote data acquisition has been demonstrated using the MXI-4 PCI bus extender. Off-the-shelf digitizers have also been used to provide real time measurements with higher bandwidth than earlier available for halo currents and loop voltages. Real time median filtering of the divertor tile currents to the outer divertor provides a signal to the control system for the divertor temperature without ELM excursions. Impurity gas puffing has been successfully introduced as a means of limiting the power load to the divertor tiles by using the divertor temperature as a feedback signal.

A text based open source software development environment and in house designed data acquisition components are currently favored as the building blocks for data acquisition and control systems in most fusion laboratories. A data acquisition system with real time signal processing and communication capability is also possible with commercial off-the-shelf components and LabVIEW RT. The experience gathered in building these systems indicates that the graphical development environment of LabVIEW is an interesting alternative for the addition of real time plasma diagnostics to a real time network in a multi-platform tokamak data acquisition and control environment.

\section{References}

[1] Developing real-time control for the world's largest telescope using NI LabVIEW with multicore functionality. URL http://sine.ni.com/cs/app/doc/p/id/cs-11465

[2] CERN uses NI LabVIEW software and PXI hardware to control world's largest particle accelerator. URL http://sine.ni.com/cs/app/doc/p/id/cs-10795

[3] M.Mitri, D.Nicolai, O.Neubauer, H.T.Lambertz, L.Schmidt, A.Khilchenko, B.Schweer, U.Maier, U.Samm, Optimised plasma stabilisation at TEXTOR with an advanced, real-time digital control scheme, Fus. Eng. Design.

URL doi : 10.1016/j.fusengdes . 2009.02.039

[4] W.Treutterer, L.Giannone, K.Lüddecke, G.Neu, G.Raupp, D.Zasche, T.Zehetbauer, Real time diagnostic integration with the ASDEX Upgrade control system, Fus. Eng. Design. URL doi : 10.1016/j.fusengdes . 2008.12.026

[5] K.Behler, H.Blank, A.Buhler, R.Cole(UCS), R.Drube, K.Engelhardt, H.Eixenberger, A.Lohs, K.Lüddecke(UCS), U.Mszanowski, R.Merkel, G.Neu, G.Raupp, W.Treutterer, M.Zilker and The ASDEX Upgrade Team, Real-Time Standard Diagnostic for ASDEX Upgrade, This conference.

[6] EasyXML for LabVIEW. URL http: //jkisoft. com/easyxml/

[7] K.H.Kim, C.J.Ju, M.K.Kim, M.K.Park, J.W.Choi, M.C.Kyum, M.Kwon, the KSTAR Control Team, Fusion Eng. Design 81 (2006) 1829.

[8] Data sheet - VMIC 5565 Reflective Memory Family. URL http: //www.gefanucembedded. com/products/resources/2136.

[9] K.Lackner, Computation of ideal MHD equilibria, Comput. Phys. Commun. 12 (1976) 33.

[10] L.Giannone, W.Schneider, P.J.McCarthy, A.C.C.Sips, W.Treutterer, K.Behler, T.Eich, J.C.Fuchs, N.Hicks, A.Kallenbach, M.Maraschek, A.Mlynek, G.Neu, G.Pautasso, G.Raupp, M.Reich, K.H.Schuhbeck, J.Stober, F.Volpe, T.Zehetbauer, M.Cerna, T.Debelle, B.Marker, S.McCaslin, M.Munroe, L.Wenzel and The ASDEX Upgrade Team, Real time magnetic field and flux measurements for tokamak control using a multi-core PCI express system, Fusion Eng. Design. 84 (2009) 825.

URL doi:10.1016/j.fusengdes . 2008.12.059

[11] J.D.Jackson, Classical Electrodynamics, p. 142, Wiley, 1999

[12] W.Schneider, P.J.McCarthy, K.Lackner, O.Gruber, K.Behler, P.Martin, R.Merkel, ASDEX Upgrade MHD equilibria reconstruction on distributed workstations, Fusion Eng. Design 48 (2000) 127.

[13] P.J.McCarthy, Analytical solutions to the Grad-Shafranov equation for tokamak equilibrium with dissimilar source functions, Physics of Plasmas 6 (1999) 3554.

[14] DXH510 PCI Express host adapter URL http: //www.dolphinics.com/products/pent-dxseries-dxh510.htm

[15] A.Vrancic, L.Wenzel, Poster at High Performance Embedded Computing 2008.

URL http://www . 11.mit.edu/HPEC/agendas/proc08/Day3/C7-Vrancic-P

[16] P.Thoman, Multigrid methods on GPU's, VDM Verlag Dr. Müller, 2008

[17] J.R.Ferron, M.L.Walker, L.L.Lao, H.E.St.John, D.A.Humphreys and J.A.Leuer, Real time equilibrium reconstruction for tokamak discharge control, Nuc. Fusion 36 (1998) 1055

[18] K.F.Mast, J.C.Vallet, C.Andelfinger, P.Betzler, H.Kraus, G.Schramm, A low noise highly integrated bolometer array for absolute measurement of VUV and soft X-ray radiation, Rev. Sci. Instrum. 52 (1991) 744.

[19] L.Giannone, D.Queen, F.Hellmann, J.C.Fuchs, Prototype of a 
radiation hard resistive bolometer for ITER, Plasma Phys. Control. Fusion 47 (2005) 2123

[20] L.Giannone, K.F.Mast, M.Schubert, Derivation of bolometer equations relevant to operation in fusion experiments, Rev. Sci. Instrum. 73 (2002) 3205.

[21] R.Neu, R.Dux, A.Kallenbach and The ASDEX Upgrade Team, Plasma operation with high-Z environment, J. Phys.: Conf. Ser. 100 (2008) 062001.

[22] G.Haas, H.Bosch, In vessel pressure measurement in nuclear fusion experiments with ASDEX gauges, Vacuum 51 (1998) 39.

[23] A.Scarabosio, G.Haas, H.Müller, R.Pugno, M.Wischmeier and The ASDEX Upgrade Team, Measurements of neutral particle fluxes under different plasma and divertor regimes in ASDEX Upgrade, Journal of Nuclear Materials 390-391 (2009) 494.

[24] A.Kallenbach, R.Dey, R.Dux, J.C.Fuchs, L.Giannone, A.Herrmann, H.W.Müller, R.Neu, T.Pütterich, V.Rohde, W.Treutterer and The ASDEX Upgrade Team, Divertor power load feedback with nitrogen seeding in ASDEX Upgrade (2009).

URL To be published, PPCF, 2010

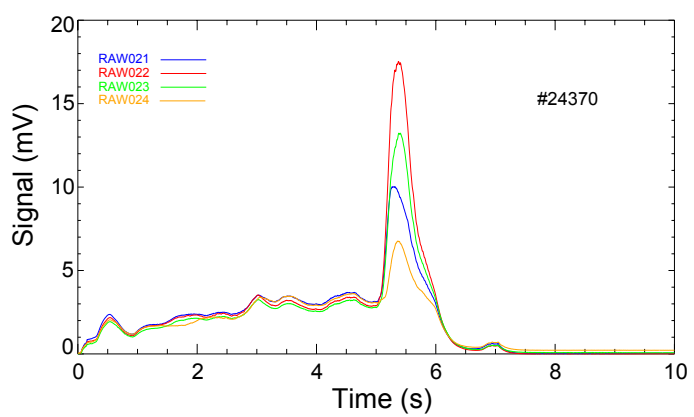

Figure 1: Time evolution of bridge voltage of Wheatstone bridge circuit of 4 bolometer channels.

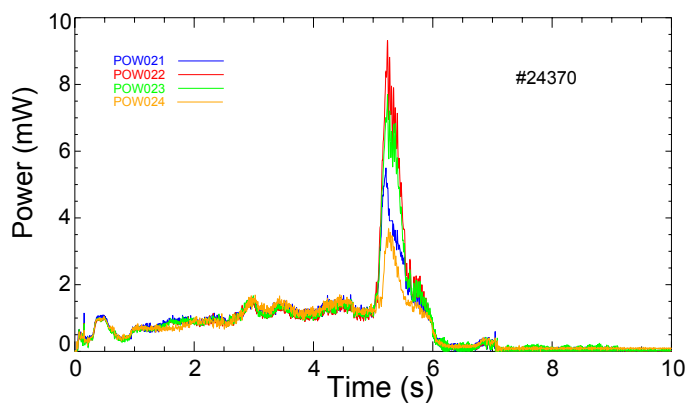

Figure 2: Time evolution of power flux of 4 bolometer channels calculated in real time using the FPGA.

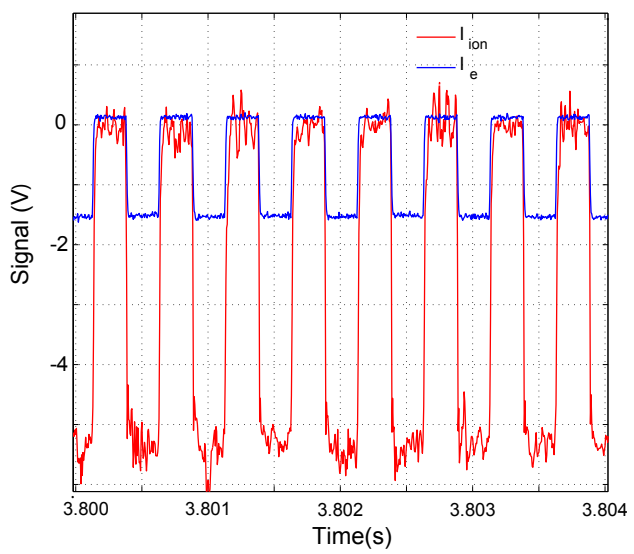

Figure 3: Time evolution of the raw signal at the ADC of the electron (blue) and ion (red) current of an ionization gauge. Modulation of the currents is due to switching of grid potentials. Demodulation for subtraction of the background values is carried out in real time on the FPGA. The magnitude of the electron current is $200 \mu \mathrm{A}$ and the magnitude of the ion current is $2.5 \mu \mathrm{A}$. 

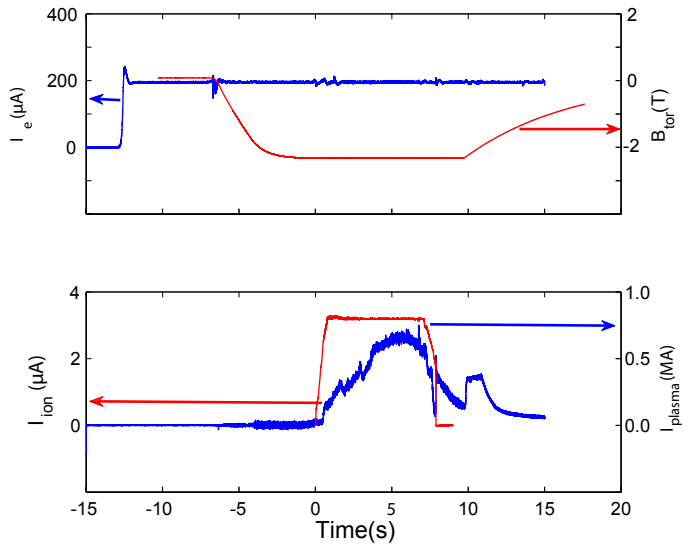

Figure 4: Overview of time evolution of electron (upper diagram blue) and ion (lower diagram - blue) current of an ionization gauge during a discharge. The magnetic field (upper diagram - red) influences the PID control of the electron current with a set value of 200 $\mu A$. The output voltage for regulating the heating filament current is calculated in real time by the FPGA library module. The plasma current (lower diagram - red) is also shown. The ion current signal after 10 s responds to a diagnostic deuterium gas puff.
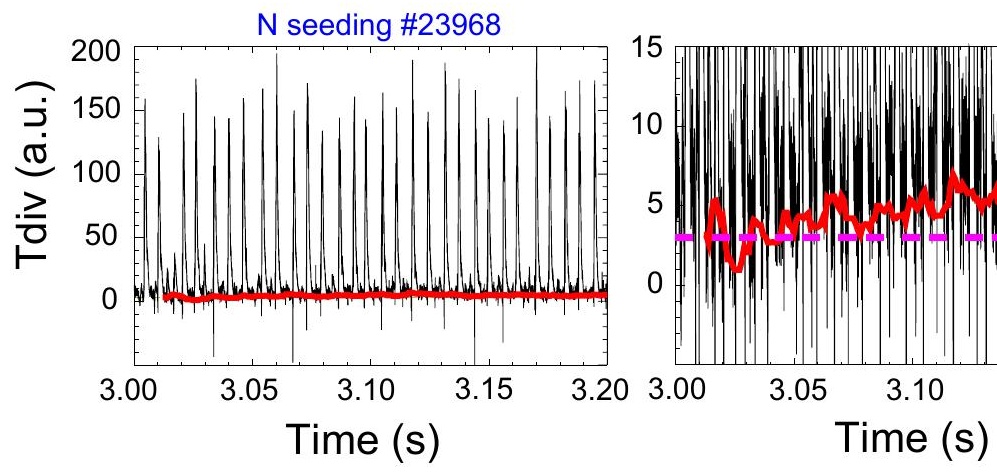

Figure 5: The divertor tile currents are sampled at $100 \mathrm{kHz}$ and the divertor temperature is proportional to the outer divertor tile current. In the left diagram, the outer divertor halo current measurement (black) and ELM free current calculated in real time by the median filter selection of the 100th largest value of the previous 700 points at 1 millisecond intervals (red) are shown. In the right diagram, the ELM free current (red) is feedback regulated by nitrogen gas puffing to the set value of 3 (dashed purple). 